cover both Neisseria gonorrhoeae (NG) and Chlamydia trachomatis (CT). The syndromic approach leads to both over-treatment (as most patients are not dually infected) and undertreatment (as asymptomatic infections are not detected). We studied the performance of the GeneXpert ${ }^{\circledR}$ nucleic acid amplification test (NAAT) as a testing platform for NG and CT in a study of the aetiology of GDS in Zimbabwe.

Materials and methods In an ongoing study, we enrolled 400 patients with GDS in 6 regionally diverse clinics in Zimbabwe serving high numbers of STI cases. Urine (men) and vaginal (women) specimens were taken for testing on 3 NAAT platforms: GeneXpert ${ }^{\circledR}$, Probetec $^{\mathrm{TM}}$ and multiplex polymerase chain reaction (M-PCR), conducted in different laboratories. We analysed overall concordance of results between platforms and assessed the performance of the GeneXpert ${ }^{\circledR}$ test when compared to a gold standard comprised of concordant results on both Probetec ${ }^{\mathrm{TM}}$ and M-PCR platforms.

Results To date, a total of 197 men and 200 women with GDS have been enrolled. Testing on all 3 platforms is complete for the 136 GDS patients enrolled in Harare. Concordance of positive or negative results for all 3 platforms was 133/136 (97.8\%) for NG and 132/136 (97.0\%) for CT. Sensitivity of the GeneXpert ${ }^{\circledR}$ platform compared to the Probetec ${ }^{\mathrm{TM}} / \mathrm{M}-\mathrm{PCR}$ combined gold standard was $100 \%$ for both NG and CT; specificity was $98.8 \%$ for NG and $100 \%$ for CT.

Conclusions Aetiologic diagnosis has long been out of reach for many countries. However, the increasing presence of nucleic acid amplification test (NAAT) devices in many countries, such as GeneXpert ${ }^{\circledR}$, for the diagnosis of tuberculosis and other infections, opens the possibility to use them for the diagnosis of other pathogens including NG and CT as an alternative or adjunct to syndromic management.

Disclosure of interest statement Nothing to declare.

\section{P09.22 THE AETIOLOGY OF GENITAL DISCHARGE SYNDROMES IN ZIMBABWE}

${ }^{1} \mathrm{~A}$ Machiha*${ }^{*},{ }^{1} \mathrm{O}$ Mugurungi, ${ }^{2} \mathrm{M}$ Tshimanga, ${ }^{3} \mathrm{P}$ Kilmarx, ${ }^{1} \mathrm{M}$ Mungati, ${ }^{1} \mathrm{f}$ Nyakura, ${ }^{2} \mathrm{G}$ Shambira, ${ }^{3} \mathrm{E}$ Gonese, ${ }^{3} \mathrm{~A}$ Herman-Roloff, ${ }^{4} \mathrm{~V}$ Kupara, ${ }^{5} \mathrm{D}$ Lewis, ${ }^{6} \mathrm{H}$ Handsfield, ${ }^{7} \mathrm{C}$ Rietmeijer. 'Ministry of Health and Child Care, Harare, Zimbabwe; ${ }^{2}$ University of Zimbabwe, Department of Community Medicine, Harare, Zimbabwe; ${ }^{3}$ US Centers for Disease Control and Prevention, Harare, Zimbabwe; ${ }^{4}$ ZICHIRE, Harare, Zimbabwe; ${ }^{5}$ The University of Sydney, Western Sydney Sexual Health, Sydney, Australia; ${ }^{6}$ University of Washington, Seattle, USA; ${ }^{7}$ Rietmeijer Consulting, Denver, USA

\subsection{6/sextrans-2015-052270.406}

Background In many countries, sexually transmitted infections (STI) are treated syndromically. Thus, male and female patients with genital discharge syndromes (GDS) receive antimicrobials covering Chlamydia trachomatis (CT), Neisseria Gonorrhoeae (NG) and Trichomonas vaginalis (TV) infections and bacterial vaginosis (BV) among women. However, periodic surveys into the aetiology of GDS are necessary to inform treatment guidelines.

Methods For this study, we enrolled 400 patients with GDS at 6 regionally diverse clinics in Zimbabwe. To date, test procedures have been completed for patients enrolled at the Harare study sites $(N=137)$. Sites were visited sequentially by a mobile unit of 3 trained nurses to enrol patients with STI syndromes, including GDS. STI history and risk data were collected by questionnaire and uploaded to a web-based database. Urine and vaginal specimens were taken for testing with a validated multiplex polymerase chain reaction assay (M-PCR, National Institute of
Communicable Diseases, Johannesburg) for CT, NG, TV and Mycoplasma genitalium (MG). Smears for Gram stain and subsequent assessment using Nugent criteria for the diagnosis of BV were obtained from all women with vaginal discharge.

Results M-PCR testing is complete for all GDS patients enrolled in Harare; 68 men and 69 women. Positivity rates were as follows. Men: $\mathrm{NG}=60.3 \%, \mathrm{CT}=13.2 \%, \mathrm{TV}=4.4 \%, \mathrm{MG}=$ 2.9\%. Women: $\mathrm{NG}=18.8 \%, \mathrm{CT}=8.7 \%, \mathrm{TV}=14.5 \%, \mathrm{MG}$ $=7.3 \%$. Among women, $31.9 \%$ met Nugent criteria for BV and $20.0 \%$ had yeast infection identified on Gram stain.

Conclusions In this preliminary analysis, gonorrhoea was the most common cause of GDS among both men and women, but much more common among men. Chlamydia infections were substantially less common among both men and women and trichomoniasis was more common than chlamydia infections among women. Regardless of investigated microbiologic causes, many women met criteria for BV. M. genitalium infections were uncommon overall. These findings will inform development of future syndromic STI management guidelines.

\section{P09.23 HIGH PREVALENCE OF HIV INFECTION AMONG PATIENTS WITH STI SYNDROMES IN ZIMBABWE: IMPLICATIONS FOR PREVENTION}

${ }^{1} \mathrm{M}$ Mungati ${ }^{*},{ }^{1} \mathrm{~A}$ Machiha, ${ }^{2} \mathrm{M}$ Tshimanga, ${ }^{3} \mathrm{E}$ Gonese, ${ }^{1} \mathrm{~J}$ Nyakura, ${ }^{3} \mathrm{P}$ Kilmarx, ${ }^{4} \mathrm{G}$ Shambira, ${ }^{4} \mathrm{~V}$ Kupara, ${ }^{3} \mathrm{~A}$ Herman-Roloff, ${ }^{5} \mathrm{~A}$ Nichol, ${ }^{6} \mathrm{H}$ Handsfield, ${ }^{7} \mathrm{C}$ Rietmeijer. ${ }^{1}$ Ministry of Health and Child Care, Harare, Zimbabwe; ${ }^{2}$ University of Zimbabwe, Department of Community Medicine, Harare, Zimbabwe; ${ }^{3}$ US Centers for Disease Control and Prevention (CDC), Harare, Zimbabwe; ${ }^{4}$ ZICHIRE, Harare, Zimbabwe; ${ }^{5}$ Denver Health and Hospital, Denver, USA; ${ }^{6}$ University of Washington, Seattle, USA; ${ }^{7}$ Rietmeijer Consulting, Denver, USA

\subsection{6/sextrans-2015-052270.407}

Introduction The occurrence of sexually transmitted infection (STI) syndromes among persons with HIV infection indicates the presence of high-risk behaviours and biological co-factors favouring HIV transmission. We determined HIV prevalence among patients enrolled in a study of the aetiology of STI syndromes in Zimbabwe.

Methods In an ongoing study, we enrolled men and women with genital discharge syndrome (GDS) or genital ulcer disease (GUD) at 6 geographically diverse clinics in Zimbabwe. We used Xpert ${ }^{\circledR} \mathrm{CT} / \mathrm{NG}$ to determine the presence of Neisseria gonorrhoeae (NG) and Chlamydia trachomatis (CT) in urethral or vaginal specimens from all study participants. Blood samples were collected for HIV testing by a standard rapid HIV test algorithm (First Response $^{\mathrm{TM}}$ followed by Alere HIV 1/2 ${ }^{\mathrm{TM}}$ ) and considered positive when reactive on both. Blood samples were also tested for treponemal antibodies (SD Bioline HIV/syphilis DUO ${ }^{\mathrm{TM}}$ ).

Results To date we completed laboratory test on 371 patients. Of these, 150 (40.4\%) were HIV-infected. HIV rates were as follows. Female GUD: $35 / 62$ (56.5\%) vs. female GDS: 46/116 $(39.7 \%, \mathrm{p}=0.05)$ and male GUD: 32/72 (44.4\%) vs. male GDS (37/121 (30.6\%, p < 0.05). HIV-infected GDS/GUD patients were significantly more likely to have positive treponemal tests compared to HIV uninfected $(10.3 \%$ vs. $4.8 \%$; p < 0.05$)$. They were also more likely to test positive for NG (40.0\% vs. $29.4 \%$; $\mathrm{p}=0.07)$, but less likely to test positive for CT $(12.2 \%$ vs. 20.3\%; $\mathrm{p}=0.07$ ).

Conclusion In our study, HIV prevalence was high among patients with STI syndromes and higher among patients with GUD than patients with GDS. The high prevalence of NG infections and evidence for recent syphilis infection among persons 
with documented HIV infection indicates high risk behaviours related to HIV acquisition and/or ongoing HIV transmission. Clinics serving patients with STI syndromes in Zimbabwe and countries with similar HIV/STI epidemiology are of continued importance in HIV diagnosis and prevention.

\section{P09.24 THE AETIOLOGY OF GENITAL ULCER DISEASE AND ASSOCIATION WITH HIV INFECTION IN ZIMBABWE}

${ }^{1} \mathrm{~A}$ Machiha*, ${ }^{1} \mathrm{O}$ Mugurungi, ${ }^{2} \mathrm{M}$ Tshimanga, ${ }^{3} \mathrm{P}$ Kilmarx, ${ }^{1} \mathrm{M}$ Mungati, ${ }^{1} \mathrm{~J}$ Nyakura, ${ }^{2} \mathrm{G}$ Shambira, ${ }^{3} \mathrm{E}$ Gonese, ${ }^{3} \mathrm{~A}$ Herman-Roloff, ${ }^{4} \mathrm{~V}$ Kupara, ${ }^{5} \mathrm{D}$ Lewis, ${ }^{6} \mathrm{H}$ Handsfield, ${ }^{7} \mathrm{C}$ Rietmeijer. ${ }^{1}$ Ministry of Health and Child Care, Harare, Zimbabwe; ${ }^{2}$ University of Zimbabwe, Department of Community Medicine, Harare, Zimbabwe; ${ }^{3}$ US Centers for Disease Control and Prevention, Harare, Zimbabwe; ${ }^{4}$ ZICHIRE, Harare, Zimbabwe; ${ }^{5}$ The University of Sydney, Western Sydney Sexual Health, Sydney, Australia; ${ }^{6}$ University of Washington, Seattle, USA; ${ }^{7}$ Rietmeijer Consulting, Denver, USA

\subsection{6/sextrans-2015-052270.408}

Background In many countries, sexually transmitted infections (STI) are treated syndromically. Thus, patients diagnosed with genital ulcer disease (GUD) in Zimbabwe receive antimicrobials that cover infections with Treponema pallidum (TP: benzathine penicillin), Haemophilus ducreyi (HD: erythromycin) and herpes simplex virus (HSV: acyclovir). However, periodic surveys into the aetiology of GUD are necessary to inform treatment guidelines.

Methods For this study, we recently completed enrollment of 200 patients with GUD at 6 clinics in Zimbabwe. To date, test procedures have been completed for patients enrolled at Harare clinics $(\mathrm{N}=70)$. Ulcer specimens were obtained for testing using a validated multiplex polymerase chain reaction assay (M-PCR, National Institute of Communicable Diseases, Johannesburg) for TP (primary syphilis), HD (chancroid), HSV (genital herpes) and Chlamydia trachomatis strains associated with lymphogranuloma venereum (CT-LGV). Blood samples were collected for HIV testing by a standard rapid HIV test algorithm (First Response ${ }^{\mathrm{TM}}$ followed by Alere HIV 1/2 ${ }^{\mathrm{TM}}$ ) and considered positive when reactive on both.

Results To date, M-PCR testing is complete for all 70 patients with GUD recruited from the Harare clinics (38 men and 32 women). Of these, 17 (24.2\%) were positive for HSV, 8 (11.4\%) were positive for TP, and 1 was positive for CT-LGV. No cases of chancroid were detected. The overall HIV positivity rate was 43.1\%. HIV rates were higher among patients with HSV $(62.5 \%, \mathrm{p}=0.07$, Chi Square Test) and TP $(87.5 \%, \mathrm{p}<0.01$, Fisher's Exact Test).

Conclusions Genital herpes was the most common cause of GUD in our survey, followed by primary syphilis. The association of HIV infection with HSV and TP suggests high risk for co-transmission; however some HSV ulcerations among HIVinfected patients may be the result of HSV reactivation among immunocompromised patients. Our study methods and data should be relevant for other countries using a syndromic management to STI control.
P09.25 DURATION OF SYPHILIS SYMPTOMS AT PRESENTATIONS IN MEN WHO HAVE SEX WITH MEN IN AUSTRALIA: ARE CURRENT PUBLIC HEALTH CAMPAIGNS EFFECTIVE?

1,2EPF Chow*, 'K Dutt, ' $\mathrm{G}$ Fehler, 'I Denham, 1,2 $\mathrm{MY}$ Chen, ${ }^{3} \mathrm{C}$ Batrouney, ${ }^{1} \mathrm{~J}$ Peel, 1,2TRH Read, ${ }^{1,2} \mathrm{CS}$ Bradshaw, ${ }^{1,2} \mathrm{CK}$ Fairley. 'Melbourne Sexual Health Centre, Alfred Health, Melbourne, Australia; ${ }^{2}$ Central Clinical School, Faculty of Medicine, Nursing and Health Sciences, Monash University, Melbourne, VIC, Australia; ${ }^{3}$ Victorian AIDS Council/Gay Men's Health Centre, Melbourne, Australia

\subsection{6/sextrans-2015-052270.409}

Introduction The rapid rise in syphilis has prompted a number of public health campaigns to assist men who have sex with (MSM) men recognise and present early with symptoms. This study aimed to investigate the temporal trend of the duration of self-report symptoms and titre of rapid plasma regain (RPR) among MSM with infectious syphilis in relation to these campaigns.

Methods 761 syphilis cases among MSM diagnosed at the Melbourne Sexual Health Centre (MSHC) from 2007-2013 were reviewed. Median and interquartile range (IQR) of duration of symptoms and RPR titres in each year were calculated.

Results The median duration of symptoms for MSM with primary and secondary syphilis were 9 days (IQR: 6-14) and 14 days (IQR: 7-30), respectively. The overall median titre of RPR in secondary syphilis (128; IQR: 64-256) was higher than in primary (4; IQR: 1-32) and early latent syphilis (32; IQR: 4-64). The median duration of symptoms for primary $(\mathrm{p}=0.11)$, secondary $(\mathrm{p}=0.24)$ and titre of RPR level for primary $(\mathrm{p}=$ $0.35)$, secondary $(\mathrm{p}=0.08)$, and early latent syphilis $(\mathrm{p}=0.85)$ did not change over time. A strong positive correlation was observed between duration of symptoms and RPR titre in both primary $(\mathrm{r}=0.36, \mathrm{p}<0.001)$ and secondary $(\mathrm{r}=0.16, \mathrm{p}=$ $0.05)$ syphilis.

Conclusion Public health campaigns were not associated with a significant shorter time from onset of symptoms to treatment. Either more effective campaigns or alternative strategies such as more frequent testing in MSM should be promoted to control the syphilis epidemic in Australia.

Disclosure of interest statement None.

\section{P09.26 TRENDS IN GONORRHOEA POSITIVITY BY NUCLEIC ACID AMPLIFICATION TESTING VERSUS CULTURE IN HETEROSEXUAL MEN IN VICTORIA, AUSTRALIA, 2007-2014}

${ }^{1,2} \mathrm{PK}$ Mannion, ${ }^{1,2} \mathrm{CK}$ Fairley, ${ }^{2} \mathrm{G}$ Fehler, ${ }^{1,2 \mathrm{MY}}$ Chen, ${ }^{1,2} \mathrm{CS}$ Bradshaw, ${ }^{1,2}$ EPF Chow ${ }^{*}$. ${ }^{1}$ Central Clinical School, Faculty of Medicine, Nursing and Health Sciences, Monash University, Melbourne, VIC, Australia; ${ }^{2}$ Melbourne Sexual Health Centre, Alfred Health, Melbourne, VIC, Australia

\subsection{6/sextrans-2015-052270.410}

Introduction A three-fold increase in Neisseria gonorrhoeae (gonorrhoea) notifications was reported amongst low prevalence 